\title{
Knowledge-based clinical pathway for medical quality improvement
}

Article

Accepted Version

Yang, H., Li, W., Liu, K. and Zhang, J. (2012) Knowledgebased clinical pathway for medical quality improvement. Information Systems Frontiers, 14 (1). pp. 105-117. ISSN 1572-9419 doi: https://doi.org/10.1007/s10796-011-9307-z Available at https://centaur.reading.ac.uk/32753/

It is advisable to refer to the publisher's version if you intend to cite from the work. See Guidance on citing.

Published version at: http://link.springer.com/content/pdt/10.1007\%2Fs10796-011-9307-z.pdf\#page-1

To link to this article DOI: http://dx.doi.org/10.1007/s10796-011-9307-z

Publisher: Springer

All outputs in CentAUR are protected by Intellectual Property Rights law, including copyright law. Copyright and IPR is retained by the creators or other copyright holders. Terms and conditions for use of this material are defined in the End User Agreement.

\section{www.reading.ac.uk/centaur}

\section{CentAUR}

Central Archive at the University of Reading

Reading's research outputs online 


\title{
Knowledge-based Clinical Pathway for Medical Quality Improvement
}

\author{
Hongqiao Yang ${ }^{1}$, Weizi $\mathrm{Li}^{2}$, Kecheng $\mathrm{Liu}^{2,3}$, Junping Zhang ${ }^{4}$ \\ (1) Hospital 309 of PLA, Beijing, China \\ (2) School of Management and Economics, Beijing Institute of Technology, China \\ (3) Informatics Research Centre, University of Reading, UK \\ (4) Carefx Corporation, Arizona, United States
}

Corresponding author: Weizi Li email: weizi.li@student.reading.ac.uk

\begin{abstract}
Clinical pathways have been adopted for various diseases in clinical departments for quality improvement as a result of standardization of medical activities in treatment process. Using knowledge-based decision support on the basis of clinical pathways is a promising strategy to improve medical quality effectively. However, the clinical pathway knowledge has not been fully integrated into treatment process and thus cannot provide comprehensive support to the actual work practice. Therefore this paper proposes a knowledge-based clinical pathway management method which contributes to make full use of clinical knowledge to control and optimize medical practice. We have developed a knowledge-based clinical pathway management system to demonstrate how the clinical pathway knowledge comprehensively supports the treatment process. The experiences from the use of this system show that the treatment quality can be effectively improved by the extracted and classified clinical pathway knowledge, seamless integration of patient-specific clinical pathway recommendations with medical tasks and the evaluating pathway deviations for optimization.
\end{abstract}

Key words: clinical pathway, quality improvement, knowledge-based system, decision support for pathway management, medical service quality

\section{Introduction}

Providing high quality of medical services is the ultimate goal of hospital work. The quality of medical care has increasingly become the social focus. Patients' increasing demand for high quality and low cost of medical services and hospitals' pursuit for maximized satisfaction lead the healthcare industry to focus on providing high quality healthcare services whilst lifting its productivity.

Currently, quality management methods in healthcare have been implemented for quality improvements, which evaluate medical services by final quality examinations of patients' cases such as hospital infection audits and mortality/morbidity audits, etc. (Chu 2001). This traditional quality management method has little effect on improving quality or reducing costs (Sakofsky 1996; Rutherford and Gibney 1997). In 1950s the concept of Total Quality Management (TQM) was proposed which especially emphasize on the basic and procedure quality rather than only focusing on end-product evaluation (Deming 1982; McManus 2000). This method has been proven to be far more effective in quality management. The healthcare industry starts to put emphasis on the quality of treatment process (Rosenstein 1999) rather than only evaluating the 
treatment results passively. Therefore, applying variances management continuously has become known as clinical pathways and been adopted for various diseases in many clinical departments for quality improvement (Wakamiya and Yamauchi 2009).

Clinical pathway is a knowledge-based, dynamic and collaborative process and evolved with the variance and up-to-date evidence dynamically. With the development of computer and internet technology, many activities in clinical pathways such as medication, laboratory tests and surgery operations are being supported by hospital information systems (HIS) and clinical information systems (CIS) which have already integrated into the core business of hospital. If the knowledge accumulated in these systems can be shared, communicated and integrated into every step during the execution of clinical pathways, the medical practice workflows will be well monitored. Furthermore, the treatment quality will be improved through knowledge based decision support and control.

However, the clinical pathway knowledge has not been fully integrated into the treatment process; the knowledge accumulated in current information systems has not been fully used to improve medical quality neither. Even though the intelligent decision support has been implemented into some of the hospital IT applications (Mathe et al. 2009; Lobach et al. 2007; Lenz and Kuhn 2004; Friedlin et al. 2007; Elson and Connelly 1995; Blaser et al. 2007), they can only support the certain parts of the medical practice instead of the whole treatment process. Barjis et al. (Barjis et al. 2010) propose the integration of decision models within business processes as one of the challenges in knowledge management area.

In order to meet this challenge, we propose a unified disease quality management method based on the clinical pathway and intelligent knowledge base. This research is expected to make full use of clinical knowledge to support and optimize medical processes. Firstly we extract the patterns for medical quality improvement by integrating the whole process of medical knowledge creation, use and updates into clinical pathways, based on which an architecture of intelligent clinical pathway systems is proposed. After that we elaborate the intelligent knowledge base which classifies the knowledge related to clinical pathways to provide comprehensive intelligent decision support to the treatment process. Finally, we demonstrate how the intelligent knowledge base improves the treatment quality through our knowledge based clinical pathway system which manages the classified clinical pathway knowledge to monitor and optimize medical behaviors.

\section{Clinical Pathways for Medical Quality Improvement}

A clinical pathway is a standardized treatment pattern to manage medical activities in order to improve the quality of service and optimize the use of resource. The clinical pathway, also called care plan is defined as the management plan that reflects the goals for patients and provides the sequence and timing of actions necessary to achieve these goals with optimal efficiency (Luc and Todd 2003). The benefits brought by the application of clinical pathway include greater consistency in practice, improved continuity of care, and monitored standards of care which all contribute to provide better treatment quality. When each clinical pathway is finished, the healthcare professionals will analyze and evaluate the variation of different patients' treatment results according to the standard pathway to avoid the similar variations or errors, which will further improve the quality of medical service and control the treatment cost. Therefore, implementation strategies for clinical pathways which deliver patient-specific advice at the time and place of treatment are most likely to be effective (Shiffman 1999; Grimshaw and Russell 
1993).

Besides the standard treatment pattern, clinical pathways are also kind of knowledge covering a wide range of content such as examinations, tests, drugs and surgeries which involve medication, test laboratory, and other clinical guidelines controlling the treatment activities. Therefore the computer-based decision support (e.g. reminder systems) based on these clinical knowledge can improve medical performance and pathway compliance by timely delivering relevant advices (Elson 1995; Johnston et al. 1994). This capability relies on the structured clinical data and rules that trigger appropriate recommendations (Zielstorff 1998; Lenz and Reichert 2005) Jeff Friedlin et al. establish the computerized physician order entry (CPOE) system with clinical decision support (CDS) which is the production system including rules classified by G-Care rule types and give alerts and reminders while placing medical orders (Friedlin et al. 2007). However the decision support is more needed during the whole treatment process than only for the order entry issue. The computer-based decision support on the basis of clinical guidelines and clinical pathways shows its effectiveness for continuous healthcare process quality improvement (Mathe et al. 2009; Chu 2001) but the knowledge coverage is not comprehensive enough to support and monitor all kinds of medical behaviors in the treatment, and the guidelines and pathways cannot be optimized in practice due to the separation with existing IT applications.

Integrating clinical decision support into IT applications and clinical workflow is the core precondition for successful quality improvement (Zielstorff 1998; Shiffman 1999; Elson 1995; Johnston et al. 1994; Maviglia 2003). Blaser et al. (Blaser and Kuhn 2004; Kuhn 2003; Blaser et al. 2007) develop the adapted software engineering process to achieve an IT-supported pathway aligned to the clinical workflows, which integrates patient-specific pathway information into documentation with rule-based recommendation. However, since system reengineering is time-consuming and unpractical, more flexible approach is still needed for comprehensive decision support in treatment process. Lobach et al. implement HL7 decision support service standard in order to provide a portable and scalable approach to clinical decision support that could facilitate the more extensive use of decision support systems (Lobach et al. 2007).

In this research we establish the knowledge based clinical pathway system for medical quality improvement, which provides the comprehensive decision support for clinical pathways based on elaborately classified knowledge bases covering different kinds of activities in clinical pathways. The close-loop management pattern of our system makes full use of knowledge accumulated in HIS and CIS supporting treatment activities and in turn ensuring the optimization of clinical pathways knowledge through the practical clinical data. Furthermore the SOA based architecture and standard, such as HL7, DICOM and IHE compliant services provide the flexible integration between clinical pathway knowledge and IT application, which will facilitate the extensive decision support in treatment process for better medical quality improvement.

\section{The Pattern of Knowledge based Clinical Pathway}

This section describes comprehensive intelligent decision support of clinical pathway for medical quality improvement and discusses how it could be supported with knowledge base technology. This illustrates a challenging application area of knowledge management for medical quality improvement. Our framework for knowledge based clinical pathways is depicted in Fig. 1 in which we summarize basic characteristics of this management pattern.

The knowledge based clinical pathways management operates throughout the whole process 
of medical knowledge creation, use and updates. Since clinical pathways is the evidence based knowledge, which guides the treatment process by the cooperation among different departments, the related knowledge can be extracted from the accumulated cases in HIS and CIS, which will further be optimized through its application in HIS and CIS. Therefore we present the management pattern of knowledge based clinical pathways as a close-loop pattern as showed in Fig. 1.

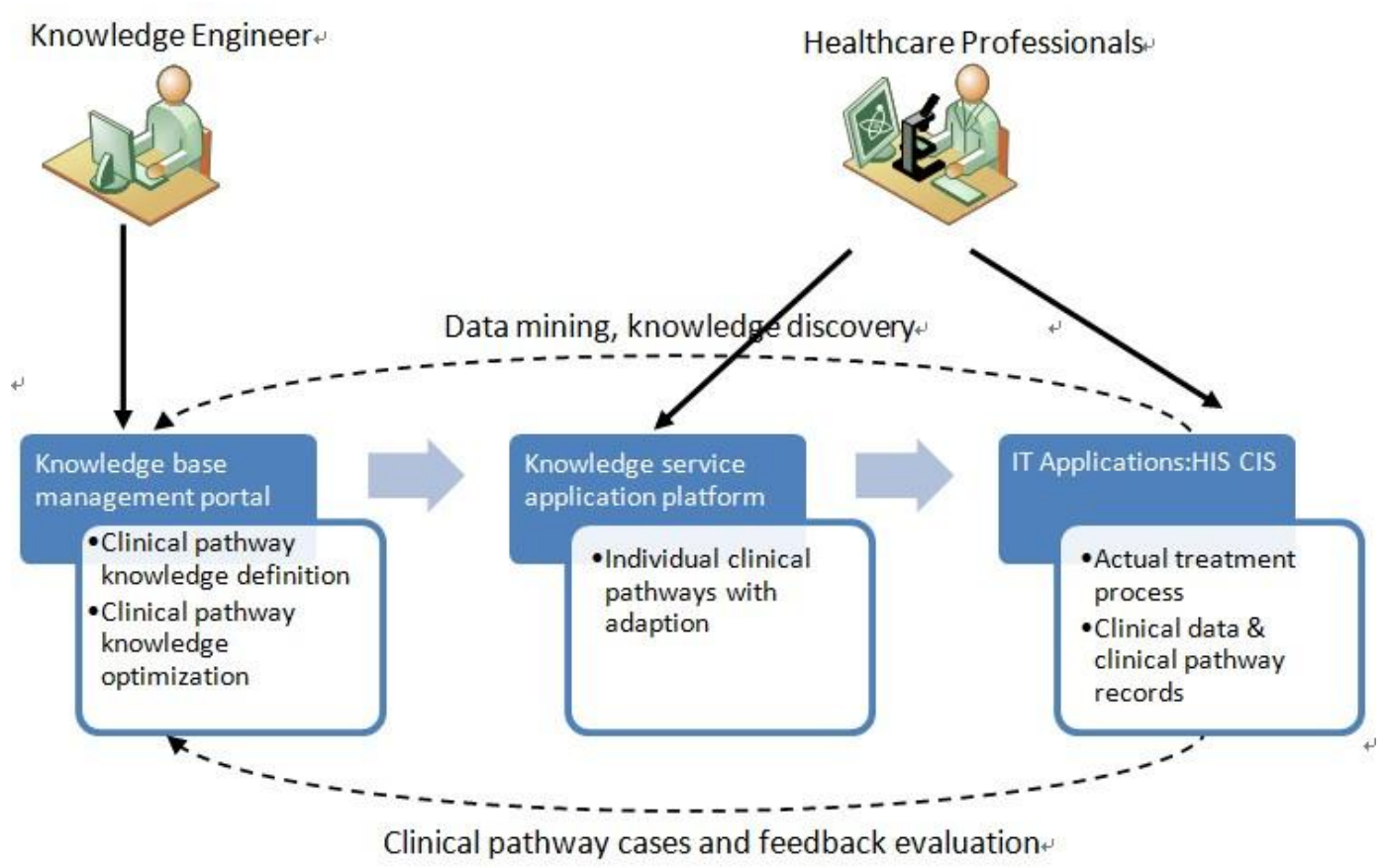

Figure 1 The Knowledge based Management Pattern of Clinical Pathways

Data stored in HIS and CIS correspond potentially to a vast source of information for supporting decisions in medical quality management and optimization (Cuggia et al. 2007). Firstly, the knowledge engineers establish the knowledge bases sorted by clinical knowledge classification through data mining and knowledge discovery from the accumulated clinical data in CIS and HIS. Furthermore, the statistical information about disease structure and the trend of main diseases in hospital will help to deeply analyze disease characteristics and support special treatments in hospital. The knowledge engineers extract and analyze the clinical data in HIS and CIS followed by defining clinical pathway knowledge in classified knowledge bases through knowledge base management portal.

The knowledge service application platform provides a comprehensive support to the whole treatment process based on the defined knowledge bases in order to standardize clinical behaviors and reduce human errors by intelligent reminders and alerts. The healthcare personnel on one hand adapt individual clinical pathways for specific patient based on defined clinical pathway knowledge bases including not only the evidence based logical sequence of clinical activities but also the related knowledge such as disease knowledge, past medical history and family heredity knowledge, allergy and drug reaction knowledge and the hospital quality management knowledge. The knowledge service application platform supports the creation of individual clinical pathways with patient-specific adaptations by applying a set of change operations and results individual 
clinical pathways deviating from the clinical pathways it was derived from.

On the other hand, the healthcare personnel execute the actual treatment process based on adapted clinical pathways with the support of HISs and CISs which are also monitored by the knowledge service application platform. However, the individual clinical pathways may be subject to change at a later point in time as well. Variations in the course of a patient treatment process are deeply inherent to medicine, and the unforeseen event is to some degree a "normal" phenomenon. Therefore in addition to the decision support for the customization of individual clinical pathways, the knowledge service application platform will also assist clinicians in dynamically adapting in-progress treatment process. For example, authorized clinicians should be allowed to add, delete, or move process steps through the platform, which is linked to HISs and CISs and contribute to the actual patient treatment process. Furthermore, the pathway deviation will be recorded by the knowledge service application platform especially for the reasons and context of a change. This enables knowledge engineers to derive optimized pathway schemes afterward and allows the reuse of knowledge about previous changes and their applications in similar situations. Therefore medical behaviors of healthcare staff and the operation of CISs and HISs are comprehensively supported and monitored by knowledge bases through knowledge service application platform. Thus the intelligent decision support can be completely integrated into each step of the whole treatment process by which the medical quality can be really improved effectively.

Realizing the full value of information and knowledge is strongly related to organizations' abilities to learn and become learning organizations (Andrew and William 1999). Clinical pathway is the evidence-based knowledge combining the standard treatment steps with long-term medical experiences. Therefore it should be improved and elaborated based on the operation in practical circumstances. The clinical pathway knowledge will be further refined and improved by continuously monitoring, analyzing and mining the change logs and learning from the applied changes, by which certain adaptions occurred repeatedly may be detected. Since some respective changes are of interest for further application, the knowledge engineers then can decide whether certain pathways need to be updated. Therefore the evaluation of clinical experiences can result in optimization for clinical pathway knowledge. The knowledge base management portal will assist knowledge engineers in analyzing and evaluating clinical record data for better treatment quality.

\section{An Architecture of Clinical Pathway Management System for Medical Quality Improvement}

Under the knowledge based clinical pathway management pattern, we propose an architecture of medical quality management systems as showed in Fig. 2. The whole system is composed of knowledge bases, knowledge base engine and hospital IT applications.

The knowledge bases are responsible for configuring and storing kinds of knowledge such as laboratory test \& examination, clinical decision and drug knowledge involved in clinical treatment as the basis for intelligent decision. Due to the dynamics lying in treatment process, the independent knowledge base management system is designed for managing and updating medical knowledge flexibly instead of the rigid knowledge module embedded in hospital IT applications.

The knowledge base engine consists of rule engine, matcher and parser for medical knowledge reasoning for the proper act according to different situation or guiding the hospital IT applications to operate for certain objectives. The knowledge base can be used by any hospital or other external applications (if authorized). For example, the physician only needs to send 
knowledge service request according to the service format defined by the connection adapter between knowledge base engine and hospital IT applications. Furthermore, the knowledge bases and engine are flexibly configured which can be loaded or uploaded dynamically and updated at any time.

Existing hospital IT applications constitute the third part of the knowledge based clinical pathway management system. Due to the heterogeneities among the existing systems in current hospital, we adopt SOA bus and connection adapter to ensure the convenient knowledge services for these systems. As Fig. 2 shows, we design the knowledge-based clinical pathway management system based on SOA open architecture, which componentizes functions as services using unified protocol and security guarantee, loose coupling interface, standardized data transformation and process automation mechanism. Furthermore the middleware of web services are constructed according to the standards such as HL7, DICOM and IHE in order to achieving information interoperability. Therefore the complex hospital IT applications and functions can be packaged and integrated seamlessly based on this architecture without reconstructing existing information systems, which preserve the original hospital investment and support the flexible medical quality management. This architecture is based on our previous work of the adaptive requirement driven architecture for integrated healthcare systems (Yang et al. 2010) which can dynamically identify and organize systems functions for users' requirement. We have also developed a detailed integration framework based on ontology and HL7 virtual database for interoperability among heterogeneous systems (Yang and Li 2009).

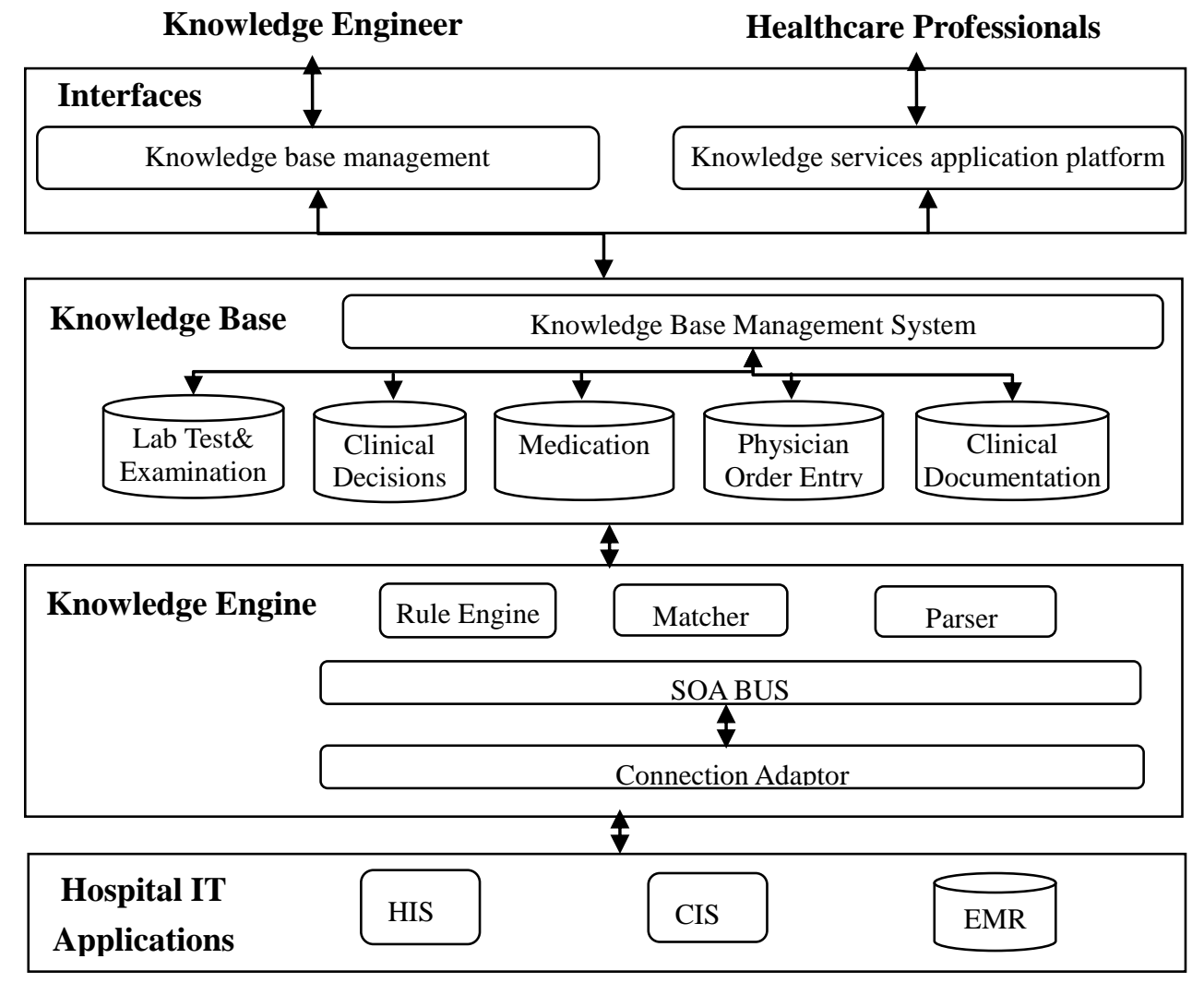

Figure 2 The Architecture of Knowledge based Clinical Pathway Management System 


\section{Knowledge-based Management System for Clinical Pathways}

The knowledge-based management system (KBMS) as shown in Fig.2 combining artificial intelligence and decision support capabilities are the core part for intelligent decision support in treatment process. The intelligent decision system is able to use human knowledge such as the descriptive knowledge for decision support, procedural knowledge for decision process and the inference knowledge for problem solution to help to solve the complex decision problems through logical reasoning. Therefore, many kinds of knowledge service applications such as intelligent decision alerts and reminders can be provided by KBMS which combine medical knowledge bases, intelligent decision system and the existing hospital IT applications. These knowledge services can help the healthcare professionals to master and manipulate related clinical knowledge and for proper practices to ensure high-quality medical services.

The KBMS in our research on one hand is responsible for clinical pathway knowledge definition, maintenance and optimization by knowledge engineers through knowledge base management portal. On the other hand it is responsible for providing intelligent decision support to healthcare professionals through knowledge service application platform as showed in Fig.2.

\subsection{The Classified Clinical Pathways Knowledge Bases}

We establish the knowledge bases by analyzing sources of clinical pathway knowledge for where we consider (1) tacit knowledge of domain experts; (2) published literature on clinical pathways; (3) existing clinical pathways in use at current hospitals; and (4) clinical data in current hospital IT applications. We have developed the modelling method of clinical pathways and the mechanism for customization ( $\mathrm{Li}$ et al. 2008, 2009, 2010). The clinical pathway knowledge is classified along 2 axes: clinical facts and clinical rules. For knowledge base management purpose, the classification of clinical pathway knowledge along these two axes ensures that the final KBMS provide a comprehensive intelligent decision support for the execution of clinical pathways.

The medical knowledge bases mainly include the healthcare professionals' knowledge such as the decision and experience knowledge for certain treatment in form of facts and rules. The facts describe all the activity concepts in a certain treatment domain and their responsible personnel and departments. In our research we classify the treatment facts into 10 main types according to the long-term treatment experience and the analysis of large amount of clinical records. These 10 types of treatment facts cover all kinds of activities involved in clinical pathways, which are as follows: (1) medication: the recommended medicine for certain disease; (2) clinical evaluation: the documentation for the evaluation of patient's condition, examination and test results; (3) nutrition: the recommended meals for the patient with certain disease; (4) observations: the documentation for patient's vital signs such as blood pressure and temperature; (5) activity: the behavior constrains for patient with certain disease such as absolute bed rest; (6) nursing: different levels of nursing and education; (7) surgery: surgery types for certain disease; (8) examination: the recommended examinations for certain diseases; (9) consultation: consultation content for certain disease; (10) variation :the record of patient condition deviate from standard pathway record. For example, the medication facts in clinical pathway for patient suffered with acute coronary syndrome include the items such as aspirin, clopidogrel, statins and other related items.

Besides the facts bases discussed above, which provide all kinds of possible medical activities involved in clinical pathways for certain diseases, the rules further specify the details of 
these activities such as when and how certain clinical activities must happen or not. Based on our research on a substantial number of clinical pathways, we classify the rules into pathway rules and activity rules. Pathway rules control the process in clinical pathways, which consist of: (1) single disease treatment indices rules: the quality indices of certain disease issued by national health department which need to be achieved through treatment. For example, a patient suffering from acute coronary syndrome (Figure 4) may have a number of progress nodes for achieving the quality management control objectives such as "Using aspirin immediately when admitted", "Performing left ventricular evaluation", "Length of stay and cost", "Ensuring the indication for aspirin ,ACEI/ARB when discharge" and other objectives; (2) pathway application criteria rules: the rules determining whether a patient is suitable for a certain pathway (3) activity steps sequences rules: the logical sequential and parallel relation among the steps of clinical pathways (4) activity step rules: rules determining the medical activities in each step (4) step reminder rules: triggering some intelligent reminder information when certain step is going to be executed (5) pathway selection rules :rules determining which branch to execute.

The activity rules refer to the detailed application conditions and constraints of classified medical activities such as medication, examination and other kinds of activities in facts bases. Since every kind of medical activities have their own indications and contradictions, the considerate decision support of that information will effectively reduce human errors and improve treatment quality. We list part of classifications of activity rules in our knowledge bases as showed in table 1. Taking medical services which have a total number of 16350 knowledge items for example, we have sorted out 6 kinds of rules that cover all the medication details such as drug-drug interaction, allergy tables and other drug usage information, which will provide intelligent decision support for doctor's prescription. The detailed knowledge classifications cover most of the clinical activities in clinical pathway so that the comprehensive intelligent reminder can be provided during treatment process. In this way, we not only manage the logical and time sequence of medical activities in clinical pathway, but also establish a set of well classified knowledge bases for them. Therefore these sets of knowledge bases can support decisions for the whole treatment process and improve medical quality more effectively.

Table 1 Example of Typical Knowledge Bases

\begin{tabular}{|l|l|}
\hline Knowledge Bases Category & $\begin{array}{l}\text { Number } \\
\text { of Items }\end{array}$ \\
\hline Medication Services & 16350 \\
\hline Drug-Drug Interactions for CPOE & 2000 \\
\hline First Databank DDIs/Allergy Tables for Pharmacy and CPOE & 4000 \\
\hline Geriatric Expert Dosing & 100 \\
\hline Renal Expert Dosing & 250 \\
\hline Pediatric and Neonatal Expert Dosing & 2000 \\
\hline Drug Dictionary with Venue/Clinical Discipline Specific Mappings & 8000 \\
\hline Other Order-Entry related Decision Support & 1230 \\
\hline Consequent Lab Display & 60 \\
\hline Consequent Orders & 60 \\
\hline Indication Screened Orders & 20 \\
\hline Antibiotic restriction/decision support & 40 \\
\hline
\end{tabular}




\begin{tabular}{|l|l|}
\hline Drug-Lab prescribing error decision support & 350 \\
\hline Drug-Problem prescribing error decision support & 700 \\
\hline Ambulatory Problem List Database & 1000 \\
\hline Physician Order Entry Knowledge Bases & 2100 \\
\hline Inpatient Order Sets/Templates & 600 \\
\hline Chemotherapy Protocols (investigational and therapeutic) & 1500 \\
\hline Surveillance and Notification Alerts & 230 \\
\hline Pharmacy ADE \& Potential ADE notification alerts & 80 \\
\hline Physician ADE/potential ADE notification alerts- inpatient & 20 \\
\hline Physician Result Notification Handling Alerts- outpatient & 100 \\
\hline Health Maintenance and Preventive Reminders & 30 \\
\hline Physician Order Entry Knowledge Bases & \\
\hline Ambulatory Order Templates & $200+$ \\
\hline Specialty and Disease-Based Filters/Dashboards & 100 \\
\hline Specialty Views of Data & $50+$ \\
\hline Clinician Population-Management Dashboards & $50+$ \\
\hline Clinical Documentation Templates & 1000 \\
\hline In-Patient Documentation & $500+$ \\
\hline Outpatient Documentation & $500+$ \\
\hline
\end{tabular}

\subsection{Inference Engine for Clinical Pathway}

The inference engine defines clinical decisions using predefined medical facts and rules knowledge, which separates the medical decision logic from hospital IT applications codes, so that the decision support can be applied for more extensive use and flexibly integrated into the treatment process with lower cost for application maintenance through reducing the complexity of the business process logics.

There are two kinds of inference pattern: forward chaining and backward chaining. Forward and backward chaining are reciprocal strategies for reasoning about one part of a decision on the basis of what is known or conjectured about other parts in the decision(Darden 2002). Forward chaining uses the early stages of a decision to reason about the types of entities and activities that are likely to be found downstream. It is driven by data and executed by one or more rules. When there are more than one matched rules, the agenda will execute the predefined activities until all the actions are finished. However, backward chaining reasons from the entities and activities in afterward stages in a decision to find entities and activities appearing earlier, which is driven by a hypothetical goal and try to find proper results. If no matched rules can be found then another sub goal will be set to look for proper rules until all the results are found or no sub goals can be found.

The process of clinical decision can be nicely illustrated by forward chaining and backward chaining. The information of patient state and treatment progress in clinical pathway can trigger related rules and provide intelligent decision reminder by forward chaining. However the execution of clinical pathway needs to achieve single disease quality indices such as disappearance of certain symptoms, length of stay and treatment cost which can only be achieved by backward chaining. Therefore we adopt the inference mechanism combined with forward chaining and backward chaining in medical knowledge base for decisions in the treatment process. 
Due to the complexity of matching algorithm of our knowledge bases, we simply list the main steps as follows: (1) input initial data goals into working memory (2) compare the initial data and goals with the rules through matcher and parser engine (3) if more than one rules are triggered then put these rules into conflict set (4) put the triggered rules into agenda to solve the confliction (5) execute the rules in agenda.

\section{Knowledge-based Clinical Pathway System for Quality Improvement}

In order to provide the intelligent decision support to clinical pathway management for medical quality improvement, we have established detailed classified knowledge bases and developed a knowledge-based clinical pathway system which is implemented according to the knowledge-based clinical pathway pattern we proposed above. In this section we describe our experiences with utilizing this system to implement clinical pathway for patients with acute coronary syndrome at the department of cardiology, the Hospital 309 of PLA, China.

\subsection{Project Background}

The care process for patients with acute coronary syndrome at the department of cardiology, Hospital 309 of PLA was analyzed. The weak points were identified such as medical errors, adverse events, unnecessary examinations or tests, insufficient documentation quality and delays in length of stay through data mining and knowledge discovery based on accumulated clinical data in hospital IT applications. Furthermore, the research on published guidelines, clinical pathways and medical practices was performed. The medical knowledge engineers establish classified knowledge bases elaborately and the target pathway was defined through the consensus process among healthcare professionals. Fig. 3 shows a simplified diagram of the pathway. For each step in the pathway there exists a detailed description with recommendations, e.g. for examinations or appropriate medication.

Therefore the knowledge based clinical pathway system and its integration into hospital IT applications in the Hospital 309 of PLA (e.g. doctor workstations and nurse workstations) is developed with the aim of getting active decision support at the time and place during the execution of clinical pathway to improve treatment quality. This system is established based on the cooperation with Carefx cooperation, whose healthcare IT solution is used in more than 600 hospitals, health systems, regional health information organizations (RHIOs), and health information exchanges (HIEs) across North America and Europe to help healthcare organizations advance the quality and safety of patient care delivery. 


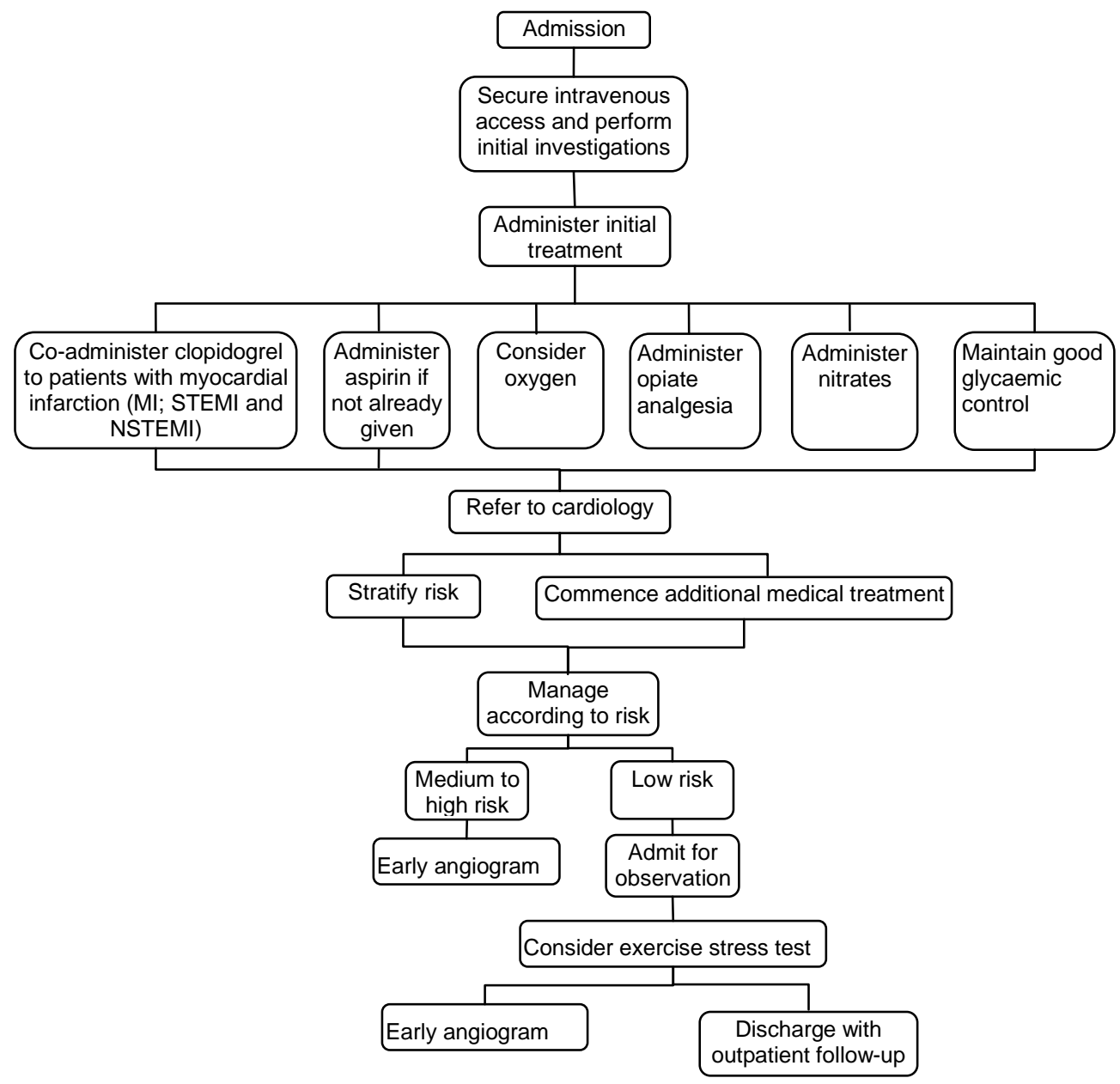

Figure 3 Clinical pathway for acute coronary syndrome

\subsection{Clinical pathway knowledge management}

The classified clinical pathways knowledge resulted from data mining and knowledge discovery of the clinical records, health professionals' experiences and related domain knowledge are specified in form of pathway, activity rules and structured medical activities for different goals (Yasnoff and Miller 2003). Pathway and activity rules describe the recommended clinical pathways process, indications and contradictions. Structured medical activities describe the medical activities of each step. All of the information are sorted and stored as classified content of knowledge bases as discussed in 5.1. Furthermore, they are represented in kinds of forms such as table, rule and flowchart for different goals

Fig. 4 shows the knowledge base management portal controlled by medical knowledge engineers for clinical pathway configuration. We discuss basic forms referring to the numbers in Fig. 4 which consists of 8 forms of related information (as annotated $1-8$ in the figure): (1) clinical pathway process form: defining the treatment steps and pathway branches with sequential and parallel relations; (2) indices form: defining the quality indices of certain disease issued by national health department; (3) pathway evaluation form: defining the condition to enter the pathway; (4)complication: defining the associated complication information; (5) details of step: defining the medical activities including medication, evaluation, nutrition, observation, activity, 
nursing, surgery, examination and consultation in each step with further order details of each classification such as the drug varieties, drug names and usage information as showed in the bottom of figure 4; (6) clinical reminder: particular attention for certain step; (7) execution conditions: defining the condition of pathway branches; (8) merge pathway: merge the pathway of other diseases. Therefore the pathway knowledge of progress steps and kinds of medical activities in each step, together with the knowledge such as order entry, surveillance alerts and other clinical decision support information are stored in classified knowledge bases for further decision support.

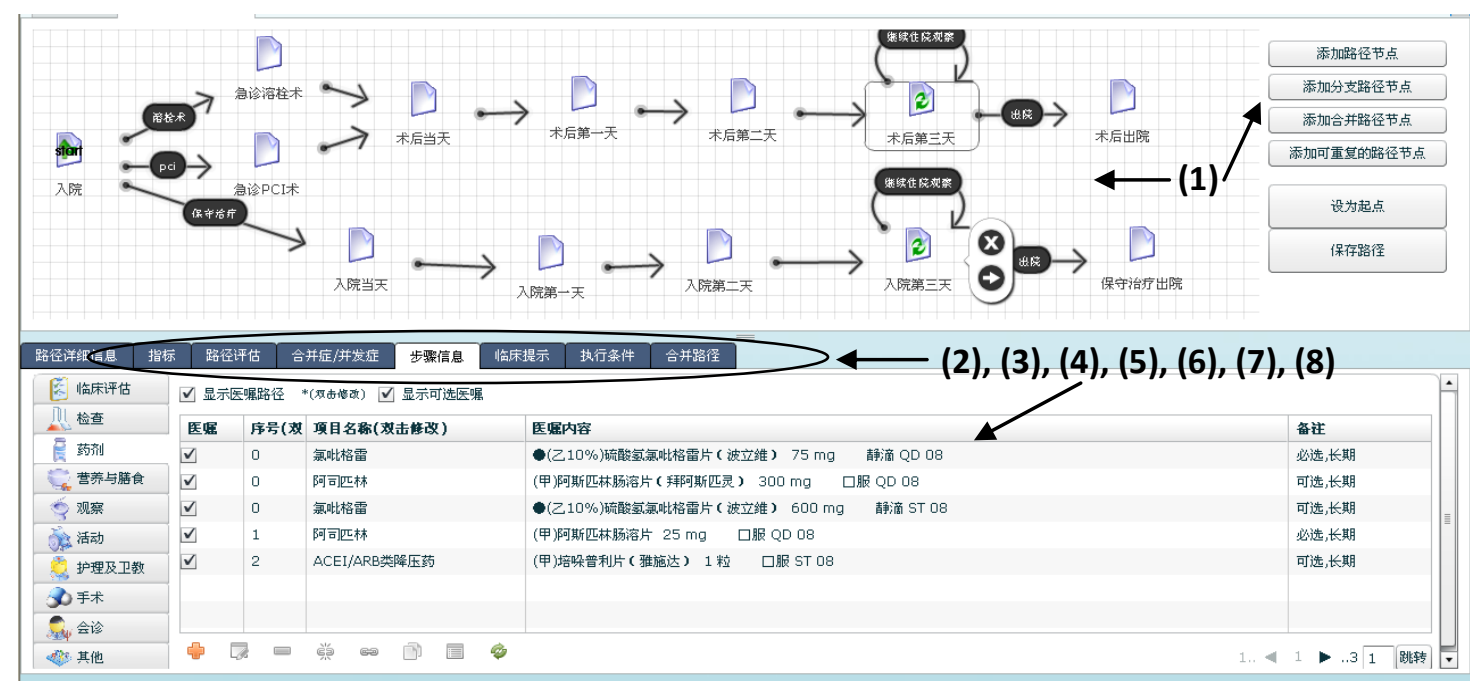

Figure 4 Knowledge Base Management Portal for Clinical Pathway Configuration

\subsection{Knowledge based clinical pathway for medical quality control}

The clinical pathway system controls the medial service quality through monitoring doctors' medical behaviors based on the knowledge base. The medical quality control is automatically activated whenever a patient starts to be treated through the pathway. Fig. 5 shows the knowledge service application platform for clinical pathway management of acute coronary syndrome. All the progress steps, different kinds of medical activities and other related clinical knowledge for this disease have been defined in knowledge bases, which will monitor and standardize hospital personnel's medical behaviors in the treatment.

As Fig. 5 shows, patient-specific information for different kinds of medical activities details in the first step of acute coronary syndrome pathway is given by a combination of various forms of decision support including implicit(structured data entry) and explicit reminders(plausibility checks, alerts, dash boards). Medical activities corresponding to the existing pathway recommendation are listed and additionally marked with a checkbox for selection. Deviation from the pathway is possible but needs to be documented for reasons.

As an example in Fig. 5, recommendations for appropriate drugs and doses are shown for acute coronary syndrome. The red ones are compulsory which means the doctors must give reasons for not choosing them and black ones are optional. The doctor can also place orders not defined in knowledge bases with documented reasons. During the process of order data entry, the doctor can be reminded for the information of drug-drug interaction, alternative drug, allergy reaction, geriatric dosing and other intelligent support based on medication service knowledge (Table 1) defined in knowledge bases and patient's information in EPR. Therefore, treatment behaviors are controlled by the knowledge system but still have the flexibility to adapt to patient's 
situation and the recorded reasons for pathway deviation will provide decision support for further pathway optimization.

Other real time intelligent decision supports cover pathway process ,indices, kinds of medical activities in clinical pathway, such as examination, evaluation, nutrition, observation, permitted activity, nursing, surgery and consultation, as well as the order entry and other clinical documentation related decision support. The comprehensive support of our system and its seamless integration into clinical practices and IT applications are the critical way to perform medical quality control.

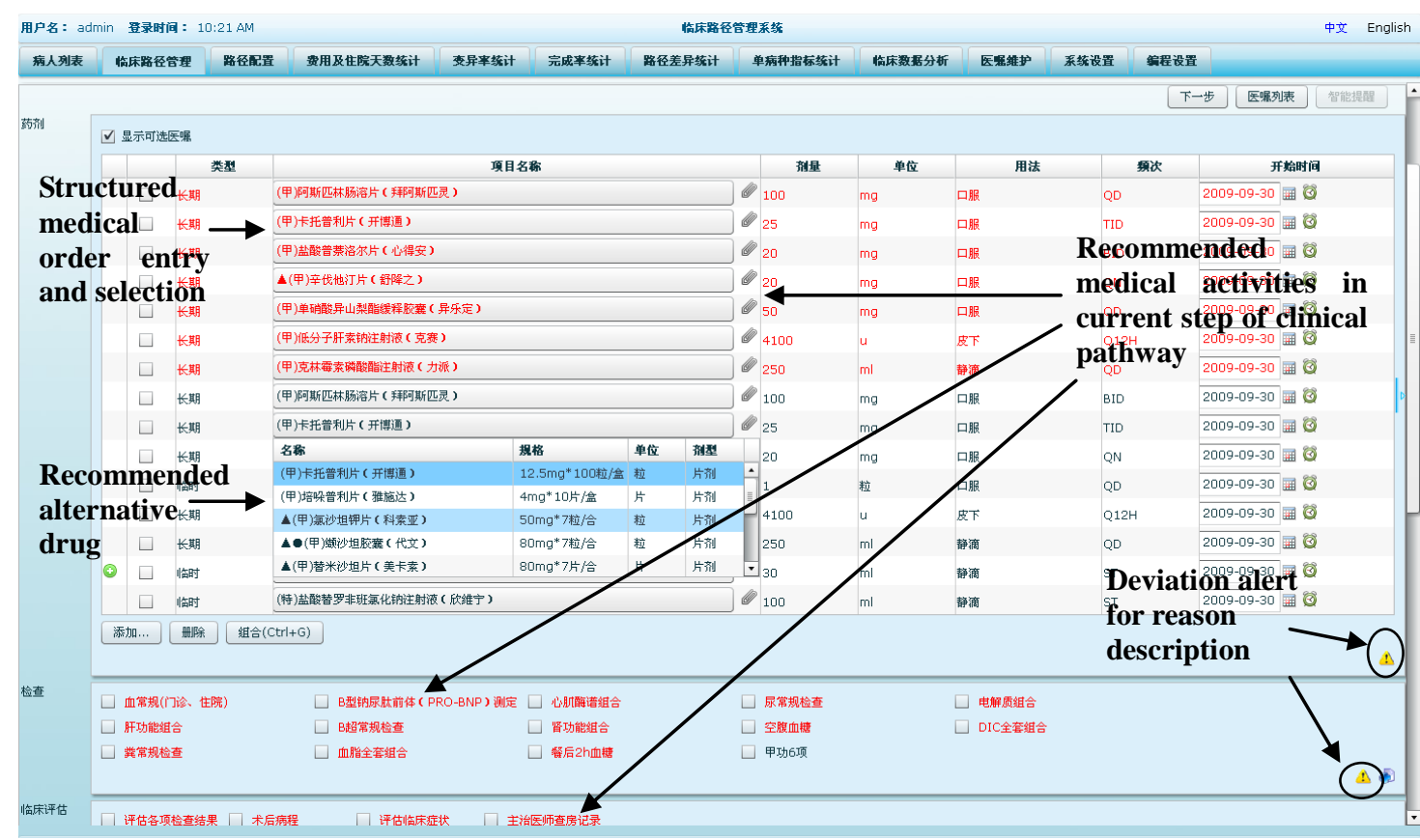

Figure 5 Knowledge Service Application Platform for Clinical Pathway Management

\subsection{Clinical Pathway Evaluation and Optimization}

One of the significant benefits of knowledge based clinical pathway management lies in the optimization of clinical pathway knowledge by evaluating pathway records in knowledge bases. The variation information for each single disease pathway is greatly important to improve treatment quality. The system provides such information by continuously monitoring, analyzing, mining and learning from the deviation logs. Therefore the medical professionals and knowledge engineers make decisions for pathway improvement through knowledge base management portal. There are four kinds of evaluation as follows: (1) cost and length of stay evaluation: evaluate the cost and length of stay of certain treatment with the average and expected level; (2) pathway completion evaluation: the percentage of pathways completion and the evaluation for the reasons of pathway failure; (3) pathway deviation evaluation: evaluate the execution status of activities in clinical pathway and analyze the reasons for deviation (4) single disease indices evaluation: evaluate the complementation status of quality indices of each pathway and analyze the reasons.

Fig. 6 shows the knowledge base management portal for pathway deviation evaluation. The system collects all the deviations through the execution status of progress steps in pathways. Besides the deviation percentage, the detailed information of pathway steps and the reasons would be displayed in a window. Highlighting any one of the deviated steps will trigger the display of the 
patient and the causes of progress failure. Therefore analysis of this information will help medical professionals and knowledge engineers to decide whether the change shall be lifted up one level by changing an existing pathway or by introducing a new one. Altogether this may result in optimized and locally tuned models for clinical pathways and produce better treatment quality.

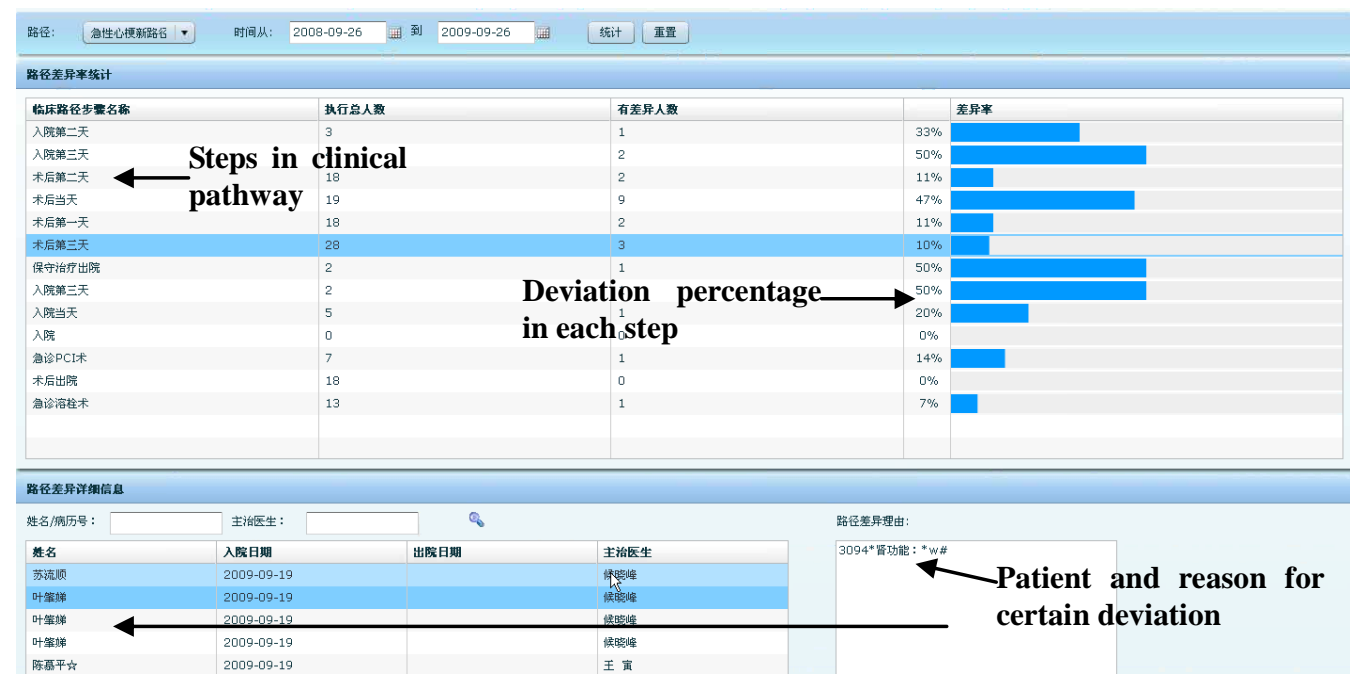

Figure 6 Knowledge base management portal for evaluation of pathway deviation

\subsection{Validation}

The clinical pathway system has been integrated into hospital IT applications such as the doctor and nurse workstations in trail operations since September 2009. 30\% of the patients with acute coronary syndrome have been administered using the system in the cardiology department, Hospital 309 of PLA. Positive feedbacks were received from clinicians using this system for intelligent support and comprehensive monitor of treatment process. 55\%-83\% medical errors and adverse events are avoided through the intelligent decision support in medical order entry; $73 \%$ of alerts affect clinicians' prescriptions; $27 \%$ of unnecessary examinations and tests are avoided through the alerts based on patients' situation and medical knowledge; $27-78 \%$ of treatments are improved through the optimization of clinical pathway based on cases evaluation. Therefore compared to the status of medical cases without decision support, the average length of stay and cost has been reduced by $31.96 \%$ and $28.46 \%$ respectively.

\section{Conclusion}

Clinical pathways have been introduced in many healthcare systems around the world with the aim of improving the quality of healthcare. The potential of decision support system based on clinical pathways to standardize medical activities, prevent medical errors and thereby improve healthcare quality is attractive. However current decision support systems can hardly support treatment process adequately. The challenges and the shortcomings of current approaches lie in the lack of full use of clinical knowledge in the treatment process. In contrast with previous clinical decision support methods, we propose the knowledge based clinical pathway management approach which makes full use of clinical knowledge to control and optimize medical behaviors. We classify the clinical pathway knowledge in details and develop the knowledge based clinical pathway management system to demonstrate how the clinical pathway knowledge can 
comprehensively support the treatment process. Our experiences from the use of this system for patient with acute myocardial infraction at Hospital 309 of PLA show that the treatment quality can be effectively improved by elaborately extracted and classified clinical pathway knowledge, seamless integration of patient-specific clinical pathway recommendations, comprehensive monitor of pathway conformance and deviation evaluation for optimization.

The implementation of the knowledge based clinical system has received positive feedbacks from users for its patient-specific clinical pathway support and the convenient, flexible and effective operation from pathway definition, execution and optimization. Our approach is continuously being evaluated and improved with expanding its knowledge bases for clinical pathway of other diseases. The knowledge based system can further provide clinical pathway oriented management through integrating into digital hospital for high quality patient-centered treatment.

\section{References}

Andrew, P. S., \& William, B. R. (1999). Information systems frontiers in knowledge management. Information Systems Frontiers, 1(3), 205-219.

Barjis, J., Gupta, A., \& Sharda, R. (2010). Knowledge work and communication challenges in networked enterprises. Information Systems Frontiers, doi: 10.1007/s10796-010-9240-6.

Blaser, R., Schnabel, M., Biber, C., Baumlein, M., Heger, O., Beyer, M., et al. (2007). Improving pathway compliance and clinician performance by using information technology. International Journal of Medical Informatics, 76, 151-156.

$\mathrm{Chu}, \mathrm{S}$. (2001). Computerised clinical pathway as process quality improvement tool. Studies in health technology and informatics, 84(Pt 2), 1135-1139.

Darden, L. (2002). Strategies for discovering mechanisms: Schema instantiation, modular subassembly, forward/backward chaining. Philosophy of Science, 69, S354-S365.

Deming, W. E. (1982). Quality, productivity \& competitive position. New York: MIT Press.

Elson, R. B., \& Connelly, D. P. (1995). Computerized decision support systems in primary care. Prim Care, 22, 365-384.

Friedlin, J., Dexter, P. R., \& Overhage, J. M. (2007). Details of a successful clinical decision support system. Paper presented at the AMIA Annual Symposium

Grimshaw, J. M., \& Russell, I. T. (1993). Effect of clinical guidelines on medical practice: A systematic review of rigorous evaluations. The Lancet, 342(8883), 1317-1322.

Johnston, M. E., Langton, K. B., Haynes, R. B., \& Mathieu, A. (1994). Effects of computer-based clinical decision support systems on clinician performance and patient outcome: A critical appraisal of research. Annals of Internal Medicine, 120, 135-142.

Kuhn, K. A., Lenz, R., Elstner, T., Siegele, H., \& Moll, R. (2003). Experiences with a generator tool for building clinical application modules. Methods Information in Medicine, 42, 37-44.

Lenz, R., \& Kuhn, K. A. (2004). Towards a continuous evolution and adaptation of information systems in healthcare. International Journal of Medical Informatics, 73, 75-89.

Lenz, R., \& Reichert, M. (2005). It support for healthcare processes. In B. B. W. van der Aalst, F. Casati, F. Curbera (Ed.), Business process management (pp. 354-363). Berlin: Springer.

Li, W., Liu, K., Li, S., \& Yang, H. (2008). Normative modeling for personalized clinical pathway using 
organizational semiotics methods. Paper presented at the International Symposium on Computer Science and Computational Technology, Shanghai, China.

Li, W., Liu, K., Li, S., \& Yang, H. (2009). An agent based approach for customized clinical pathway. Paper presented at the 2009 International Forum on Information Technology and Applications, Chengdu, China.

Li, W., Liu, K., Li, S., \& Yang, H. (2010). A semiotic multi-agent modeling approach for clinical pathway management. Journal of Computers, 5(2), 266-273.

Lobach, D. F., Kawamoto, K., Anstrom, K. J., Russell, M. L., Woods, P., \& Smith, D. (2007). Development, deployment and usability of a point-of-care decision support system for chronic disease management using the recently-approved hl7 decision support service standard. Studies in health technology and informatics, 129(Pt 2), 861-865.

Luc, K.D. , \& Todd, J. (2003). E-pathways: Computers and the patient's journey through care. Abingdon: Radcliffe Medical Press.

Cuggia, M., Rossille, D., Arnault, A., Bouget, J., \& Le Beux, P. (2007). Towards a decision support system for optimising clinical pathways of elderly patients in an emergency department. Paper presented at the Medinfo 2007: Proceedings of the 12th World Congress on Health (Medical) Informatics; Building Sustainable Health Systems, Amsterdam.

Mathe, J. L., Martin, J. B., Miller, A., Lédeczi, Á., Weavind, L. M., Nadas, A., et al. (2009). A model-integrated, guideline-driven, clinical decision-support system. IEEE Software, 26(4), 54-61.

Maviglia, S.M., R. D. Z., M. Paterno, J.M. Teich, D.W.,Bates, G.J. Kuperman (2003). Automating complex guidelines for chronic disease: Lessons learned. J. Am. Med. Inform. Assoc., 10, 154-165.

McManus, J. (2000). Quality meets process improvement. Management Services, 44(5), 14-16.

Rosenstein, A.H. (1999). Inpatient clinical decision support systems: Determining the roi. Health Financial Management, 53(2), 51-55.

Rutherford, W. E., \& Gibney, R. (1997). End-stage renal disease: A proving ground for quality improvement in health care. Seminars in Nephrology, 17(3), 218-225

Sakofsky, S. (1996). Solving the corrective action dilemma. Journal for Quality \& Participation, 19(6), 56-58.

Shiffman, R. N., Liaw, Y., Brandt, C. A., \& Corb, G. J. (1999). Computer-based guideline implementation systems: A systematic review of functionality and effectiveness. J. Am. Med. Inform. Assoc, 6, 104-114.

Wakamiya, S., \& Yamauchi, K. (2009). What are the standard functions of electronic clinical pathways? International Journal of Medical Informatics, 78, 543-550.

Yang, H., Liu, K., \& Li, W. (2010). Adaptive requirement-driven architecture for integrated healthcare systems. Journal of Computers, 5(2), 186-193.

Yang, H., \& Li, W. (2009). Modeling requirement driven architecture of adaptive healthcare system based on semiotics. Paper presented at the International Forum on Information Technology and Applications (IFITA), Chengdu, China.

Yang, H., \& Li, W. (2009). An ontology-based approach for data integration in regionally interoperable healthcare systems. Paper presented at the 11th International Conference on Informatics and Semiotics in Organisations, Beijing, China.

Yasnoff, W. A., \& Miller, P. L. (2003). Decision support and expert systems in public health In Public 
health informatics and information systems (pp. 494-512): Springer New York.

Zielstorff, R. D. (1998). Online practice guidelines: Issues, obstacles, and future prospects. Journal of the American Medical Informatics Association, 5, 227-236. 